\title{
Nonautonomous Exponential Dichotomy
}

\author{
Cornelis van der Mee
}

\begin{abstract}
In this note we generalize the strongly continuous bisemigroups generated by exponentially dichotomous operators to so-called bievolution families. These families are then related to strongly continuous bisemigroups on certain Banach spaces of continuous and measurable vector-valued functions.
\end{abstract}

Keywords. Bisemigroup, dichotomous operator, bievolution family.

\section{Introduction}

In recent years exponentially dichotomous operators $S(X \rightarrow X)$ defined on a dense linear subspace of a complex Banach space $X$ have been studied extensively $[1,7,10,12]$. They can be defined through the Laplace transform relation

$$
(\lambda-S)^{-1} x=\int_{-\infty}^{\infty} e^{-\lambda t} E(t ; x) d t,
$$

where, for each $x \in X, E(\cdot ; x): \mathbb{R} \rightarrow X$ is strongly measurable and satisfies

$$
\int_{-\infty}^{\infty} e^{\varepsilon|t|}\|E(t ; x)\|_{X} d t \leq \text { const. }\|x\|_{X}, \quad x \in X
$$

for some constant $\varepsilon>0$. Then there exists a strongly continuous function $E: \mathbb{R} \rightarrow$ $\mathcal{L}(X)$, the so-called bisemigroup, having its values in the complex Banach algebra $\mathcal{L}(X)$ of bounded linear operators on $X$ and having a strong jump discontinuity at $t=0$ such that $E(t) x=E(t ; x)$ for $0 \neq t \in \mathbb{R}$. Also $E\left(0^{+}\right)-E\left(0^{-}\right)=I_{X}$, the identity operator on $X$. Further, $\pm E\left(0^{ \pm}\right)$are complementary projections reducing $S$.

Exponentially bounded evolution families have been defined as strongly continuous operator functions $U:\left\{(t, s) \in \mathbb{R}^{2}: t \geq s\right\} \rightarrow \mathcal{L}(X)$ having the properties (i) $U(t, r) U(r, s)=U(t, s)$ for $t \geq r \geq s$, and (ii) $\|U(t, s)\|_{\mathcal{L}(X)} \leq M e^{\varepsilon(t-s)}$ for

The research leading to this note was supported by the Italian Ministry of Education and Research (MIUR) under PRIN grant no. 2006017542-003, and by INdAM-GNCS. 
certain constants $M, \varepsilon>0$. They are the natural generalizations of strongly continuous semigroups when modeling nonautonomous first order initial value problems (cf. [2] and references therein). In the context of $[4,3,2]$ exponential dichotomy pertains to the existence of a projection-valued function $P: \mathbb{R} \rightarrow \mathcal{L}(X)$ such that (i) $U(t, s) P(s)=P(t) U(t, s)$ for $t \geq s$, (ii) there exists $\varepsilon>0$ such that $\|U(t, s) P(s) x\|_{X} \leq$ const. $e^{\varepsilon(t-s)}\|P(s) x\|_{X}$ for $t \geq s$, and (iii) the restriction of $U(t, s)$ to the kernel of $P(s)$ is a boundedly invertible operator defined on the kernel of $P(t)$ with norm bounded above by const. $e^{-\varepsilon(t-s)}$ for some $\varepsilon>0$. Exponential dichotomy of exponentially bounded evolution families can be proven equivalent to the hyperbolicity of the strongly continuous semigroup $E: \mathbb{R} \rightarrow \mathcal{L}\left(L^{p}(\mathbb{R} ; X)\right)$ defined by $[E(t) f](\tau)=U(\tau, \tau-t) f(\tau-t)$ for $t \geq 0$ and $\tau \in \mathbb{R}$ (cf. [8]).

In this note we generalize the exponentially dichotomous operators as studied in $[1,12]$ to so-called bievolution families, mimicking the terminology of bisemigroups introduced in [1]. In Theorem 2.2 we prove that $U$ is a bievolution family on $X$ if and only $E$ defined by $[E(t) f](\tau)=U(\tau, \tau-t) f(\tau-t)$ is a strongly continuous bisemigroup on $C_{0}(\mathbb{R} ; X)$. In Proposition 2.1 we also show that $E_{U}$ is a strongly continuous bisemigroup on $L^{p}(\mathbb{R} ; X)(1 \leq p<\infty)$ if $U$ is a bievolution family.

Exponentially dichotomous operators have among their applications Riccati equations [10], transport equations [5], functional differential equations [9], and noncausal linear systems [6]. Some of these applications have nonautonomous counterparts conductive to treatment as bievolution systems, such as nonautonomous functional differential equations [9] and evolution equations in Banach spaces [11].

Let us introduce some notations. Given a complex Banach space $X$, we write $I_{X}$ for the identity operator on $X, \mathcal{L}(X)$ for the Banach algebra of bounded linear operators on $X, C_{0}(\mathbb{R} ; X)$ for the Banach space of strongly continuous functions $f: \mathbb{R} \rightarrow X$ such that $\|f(t)\|_{X} \rightarrow 0$ as $t \rightarrow \pm \infty$. For $1 \leq p<\infty$ we mean by $L^{p}(\mathbb{R} ; X)$ the Banach space of strongly measurable functions $f: \mathbb{R} \rightarrow X$ for which the scalar function $\|f(\cdot)\|_{X} \in L^{p}(\mathbb{R})$.

\section{Bievolution Families and Main Theorem}

Letting $\Delta_{ \pm}=\left\{(t, s) \in \mathbb{R}^{2}: \pm(t-s) \geq 0\right\}$, the disjoint (set theoretical and topological) union $\Delta=\Delta_{+} \cup \Delta_{-}$represents the Euclidean plane $\mathbb{R}^{2}$, where we distinguish between $\left(t, t^{-}\right) \in \Delta_{+}$and $\left(t, t^{+}\right) \in \Delta_{-}$. Letting $X$ be a complex Banach space, by a bievolution family on $X$ we mean a strongly continuous operator function $U: \Delta \rightarrow \mathcal{L}(X)$ having the following properties:

1. For $(t, r)$ and $(r, s)$ in $\Delta_{ \pm}$we have the product rule

$$
U(t, r) U(r, s)= \pm U(t, s) .
$$

2. For $(t, \tau) \in \Delta_{+}$and $(s, \sigma) \in \Delta_{-}$we have

$$
U(t, \tau) U(s, \sigma)=U(s, \sigma) U(t, \tau)=0 .
$$


3. There exist positive constants $M$ and $\varepsilon$ such that

$$
\|U(t, s)\|_{\mathcal{L}(X)} \leq M e^{-\varepsilon|t-s|}, \quad(t, s) \in \Delta_{ \pm} .
$$

4. We have

$$
U\left(t^{+}, t\right)-U\left(t^{-}, t\right)=I_{X}, \quad t \in \mathbb{R} .
$$

Then $U\left(t, t^{-}\right)$and $-U\left(t, t^{+}\right)$for $\left(t, t^{\mp}\right) \in \Delta_{ \pm}$are bounded complementary projections on $X$ which are strongly continuous in $t \in \mathbb{R}$.

When $U(t, s)$ only depends on $(t-s) \in \Delta$ and hence we may write $E(t-$ $s)=U(t, s)$ while distinguishing between $E\left(0^{+}\right)$and $E\left(0^{-}\right)$, we obtain a (strongly continuous) bisemigroup on $X$. The separating projection then no longer depends on $t$ and is called the separating projection of the bisemigroup. For convenience we write $\dot{\mathbb{R}}$ for the disjoint (set theoretical and topological) union of $\dot{\mathbb{R}}_{-}=(-\infty, 0]$ and $\dot{\mathbb{R}}_{+}=[0, \infty)$, so that $E$ can be viewed as a strongly continuous operator function $E: \dot{\mathbb{R}} \rightarrow \mathcal{L}(X)$.

Given a bievolution family $U: \Delta \rightarrow X$, we define the evolutionary bisemigroup $E_{U}: \dot{\mathbb{R}} \rightarrow \mathcal{L}\left(L^{p}(\mathbb{R} ; X)\right)(1 \leq p<\infty)$ or $E_{U}: \dot{\mathbb{R}} \rightarrow \mathcal{L}\left(C_{0}(\mathbb{R} ; X)\right)$ by

$$
\left(E_{U}(t) f\right)(\tau)=U(\tau, \tau-t) f(\tau-t), \quad(\tau, \tau-t) \in \Delta .
$$

Proposition 2.1. Let $1 \leq p<\infty$ and let $U: \Delta \rightarrow \mathcal{L}(X)$ be a bievolution family. Then $E_{U}: \dot{\mathbb{R}} \rightarrow L^{p}(\mathbb{R} ; X)$ is a strongly continuous bisemigroup.

Proof. Let $U: \Delta \rightarrow \mathcal{L}(X)$ be a bievolution family. For $t \in \dot{\mathbb{R}}$ we have

$$
\begin{aligned}
\left\|E_{U}(t) f\right\|_{L^{p}(\mathbb{R} ; X)} & =\left[\int_{-\infty}^{\infty}\left\|\left(E_{U}(t) f\right)(\tau)\right\|^{p} d \tau\right]^{1 / p} \\
& =\left[\int_{-\infty}^{\infty}\|U(\tau, \tau-t) f(\tau-t)\|^{p} d \tau\right]^{1 / p} \leq M e^{-\varepsilon|t|}\|f\|_{L^{p}(\mathbb{R} ; X)},
\end{aligned}
$$

which implies the boundedness of $E_{U}(t)$ for $t \in \dot{\mathbb{R}}$ as well as the exponential bound on its norm. Further, for $t, s \in \dot{\mathbb{R}}$ of the same sign we estimate

$$
\begin{aligned}
& \left\|E_{U}(t) f-E_{U}(s) f\right\|_{L^{p}(\mathbb{R} ; X)}^{p} \\
& =\int_{-\infty}^{\infty}\|U(\tau, \tau-t) f(\tau-t)-U(\tau, \tau-s) f(\tau-s)\|_{X}^{p} d \tau \\
& \leq \int_{-\infty}^{\infty}\|[U(\tau+t, \tau)-U(\tau+t, \tau+t-s)] f(\tau)\|_{X}^{p} d \tau \\
& \quad+M e^{-\varepsilon|s|} \int_{-\infty}^{\infty}\|f(\tau)-f(\tau+t-s)\|_{X}^{p} d \tau
\end{aligned}
$$

which vanishes as $s \rightarrow t$ as a result of the strong continuity of $U$. 
For $t, s \in \dot{\mathbb{R}}$ we have

$$
\begin{aligned}
& \left(E_{U}(t) E_{U}(s) f\right)(\tau)=U(\tau, \tau-t)\left(E_{U}(s) f\right)(\tau-t) \\
& =U(\tau, \tau-t) U(\tau-t, \tau-t-s) f(\tau-t-s) \\
& = \begin{cases}U(\tau, \tau-t-s) f(\tau-t-s)=\left(E_{U}(t+s) f\right)(\tau), & t, s \geq 0, \\
-U(\tau, \tau-t-s) f(\tau-t-s)=-\left(E_{U}(t+s) f\right)(\tau), & t, s \leq 0, \\
0, & t s<0,\end{cases}
\end{aligned}
$$

which implies the product rule. Next,

$$
\left(E_{U}\left(0^{+}\right) f\right)(\tau)-\left(E_{U}\left(0^{-}\right) f\right)(\tau)=U\left(\tau, \tau^{-}\right) f(\tau)-U\left(\tau, \tau^{+}\right) f(\tau)=f(\tau),
$$

so that $E_{U}\left(0^{+}\right)-E_{U}\left(0^{-}\right)=I_{L^{p}(\mathbb{R} ; X)}$. Thus, if $U: \Delta \rightarrow \mathcal{L}(X)$ is a bievolution family on $X$, then $E_{U}$ is a strongly continuous bisemigroup on $L^{p}(\mathbb{R} ; X)$.

We now derive the main result of this note.

Theorem 2.2. The operator function $U: \Delta \rightarrow \mathcal{L}(X)$ is a bievolution family iff $E_{U}: \dot{\mathbb{R}} \rightarrow \mathcal{L}(X)$ is a strongly continuous bisemigroup on $C_{0}(\mathbb{R} ; X)$.

Proof. For $t \in \dot{\mathbb{R}}$ we have

$$
\left\|E_{U}(t) f\right\|_{C_{0}(\mathbb{R} ; X)} \leq \sup _{\tau \in \mathbb{R}}\|U(\tau, \tau-t)\|_{\mathcal{L}(X)}\|f\|_{C_{0}(\mathbb{R} ; X)} \leq M e^{-\varepsilon|t|}\|f\|_{C_{0}(\mathbb{R} ; X)},
$$

which yields the boundedness of $E_{U}(t)$ and the exponential bound on its norm. For $t, s \in \dot{\mathbb{R}}$ of the same sign we estimate

$$
\begin{aligned}
& \left\|E_{U}(t) f-E_{U}(s) f\right\|_{C_{0}(\mathbb{R} ; X)} \\
& =\sup _{\tau \in \mathbb{R}}\|U(\tau, \tau-t) f(\tau-t)-U(\tau, \tau-s) f(\tau-s)\|_{X} \\
& \leq \sup _{\tau \in \mathbb{R}}\|[U(\tau+t, \tau)-U(\tau+t, \tau+t-s)] f(\tau)\|_{X} \\
& \quad+M e^{-\varepsilon|s|} \sup _{\tau \in \mathbb{R}}\|f(\tau)-f(\tau+t-s)\|_{X},
\end{aligned}
$$

proving the strong continuity of $E_{U}: \dot{\mathbb{R}} \rightarrow C_{0}(\mathbb{R} ; \mathcal{L}(X))$. Thus, if $U: \Delta \rightarrow \mathcal{L}(X)$ is a bievolution family on $X$, then $E_{U}$ is a strongly continuous bisemigroup on $C_{0}(\mathbb{R} ; X)$.

Conversely, let $E_{U}: \dot{\mathbb{R}} \rightarrow C_{0}(\mathbb{R} ; X)$ be a strongly continuous bisemigroup. For $x \in X$ and $\phi \in C_{0}(\mathbb{R})$ we define $F_{(\phi, x)} \in C_{0}(\mathbb{R} ; X)$ by

$$
\left[F_{(\phi, x)}\right](t)=\phi(t) x, \quad t \in \mathbb{R} .
$$

Then taking a function $\phi$ without zeros we see from the identity

$$
U(\tau, \tau-t) x=\frac{\left[E(t) F_{(\phi, x)}\right](\tau)}{\phi(\tau-t)}
$$


that $U: \Delta \rightarrow \mathcal{L}(X)$ is strongly continuous. For $t, s \in \dot{\mathbb{R}}$ we have on the one hand

$$
\begin{aligned}
{\left[E_{U}(t) E_{U}(s) F_{(\phi, x)}\right](\tau) } & =U(\tau, \tau-t)\left[E_{U}(s) F_{(\phi, x)}\right](\tau-t) \\
& =U(\tau, \tau-t) U(\tau-t, \tau-t-s)\left[F_{(\phi, x)}\right](\tau-t-s) \\
& =\phi(\tau-t-s) U(\tau, \tau-t) U(\tau-t, \tau-t-s) x
\end{aligned}
$$

and on the other hand

$$
\begin{aligned}
{\left[E_{U}(t+s) F_{(\phi, x)}\right](\tau) } & =U(\tau, \tau-t-s)\left[F_{(\phi, x)}\right](\tau-t-s) \\
& =\phi(\tau-t-s) U(\tau, \tau-t-s) x .
\end{aligned}
$$

Taking $\phi \in C_{0}(\mathbb{R})$ to be nonzero, we obtain from the product rule for $E_{U}$ the product properties 1 and 2 for $U$. Moreover,

$$
\phi(\tau) x=\left\{E_{U}\left(0^{+}\right)-E_{U}\left(0^{-}\right)\right\} F_{(\phi, x)}(\tau)=\phi(\tau)\left\{U\left(\tau, \tau^{-}\right) x-U\left(\tau, \tau^{+}\right) x\right\}
$$

implies that $U\left(\tau, \tau^{-}\right)-U\left(\tau, \tau^{+}\right)=I_{X}$. Finally,

$$
\begin{aligned}
\|\phi\|_{C_{0}(\mathbb{R})}\|U(\tau, \tau-t) x\|_{X} & =\left\|E_{U}(t) F_{(\phi, x)}\right\|_{C_{0}(\mathbb{R} ; X)} \\
& \leq M e^{-\varepsilon|t|}\left\|F_{(\phi, x)}\right\|_{C_{0}(\mathbb{R} ; X)}=M e^{-\varepsilon|t|}\|\phi\|_{C_{0}(\mathbb{R})}\|x\|_{X}
\end{aligned}
$$

implies the exponential decay condition 4 on $U$. Thus, $U: \Delta \rightarrow \mathcal{L}(X)$ is a bievolution family.

\section{References}

[1] H. Bart, I. Gohberg, and M.A. Kaashoek, Wiener-Hopf factorization, inverse Fourier transforms and exponentially dichotomous operators, J. Funct. Anal. 68, 1-42 (1986).

[2] C. Chicone and Yu. Latushkin, Evolution Semigroups in Dynamical Systems and Differential Equations, Math. Surveys and Monographs 70, Amer. Math. Soc., Providence, RI, 1999.

[3] Ju.L. Daleckii and M.G. Krein, Stability of Solutions of Differential Equations in Banach Space, Transl. Math. Monographs 43, Amer. Math. Soc., Providence, RI, 1974; also: Izdat. "Nauka," Moscow, 1970 (in Russian).

[4] H.O. Fattorini, The Cauchy Problem, Encycl. Math. Appl. 18, Addison Wesley, Reading, 1983.

[5] W. Greenberg, C.V.M. van der Mee, and V. Protopopescu, Boundary Value Problems in Abstract Kinetic Theory, Birkhäuser OT 23, Basel and Boston, 1987.

[6] M.A. Kaashoek, C.V.M. van der Mee, and A.C.M. Ran, Wiener-Hopf factorization of transfer functions of extended Pritchard-Salamon realizations, Math. Nachrichten 196, 71-102 (1998).

[7] M.A. Kaashoek and S.M. Verduyn Lunel, An integrability condition on the resolvent for hyperbolicity of the semigroup, J. Diff. Eqs. 112, 374-406 (1994).

[8] Yu. Latushkin and T. Randolph, Dichotomy of differential equations on Banach spaces and an algebra of weighted translation operators, Integral Equations and Operator Theory 23, 472-500 (1995). 
[9] J. Mallet-Paret and S.M. Verduyn Lunel, Exponential dichotomies and WienerHopf factorizations for mixed-type functional differential equations, J. Diff. Eqs., to appear.

[10] A.C.M. Ran and C. van der Mee, Perturbation results for exponentially dichotomous operators on general Banach spaces, J. Funct. Anal. 210, 193-213 (2004).

[11] R.J. Sacker and G.R. Sell, Dichotomies for linear evolution equations in Banach spaces, J. Diff. Eqs. 113, 17-67 (1994).

[12] C. van der Mee, Exponentially Dichotomous Operators and Applications, Birkhäuser, Basel and Boston, in preparation.

Cornelis van der Mee

Dip. Matematica e Informatica

Università di Cagliari

Viale Merello 92

I-09123 Cagliari

Italy

e-mail: cornelis@krein.unica.it

Submitted: April 24, 2007 